\title{
REGIONAL CHANGES IN BRAIN GLUCOSE METABOLISM REFLECT COGNITIVE IMPAIRMENTS IN AGED RATS ${ }^{1}$
}

\author{
FRED H. GAGE, ${ }^{2}$ PAUL A. T. KELLY, ${ }^{3}$ AND ANDERS BJÖRKLUND \\ Department of Histology, University of Lund, S-223 62 Lund, Sweden \\ Received March 1, 1984; Revised May 23, 1984; Accepted May 30, 1984
}

\begin{abstract}
Aged rats ( 22 to 24 months) and young control rats ( 3 months) were tested in a battery of behavioral tests which included tests of learning, place navigation, sensorimotor integration, motor coordination, activity, and exploration. Following testing all animals were analyzed in an unanesthetized state for their local glucose utilization. Significant differences in glucose utilization were found between the aged and young groups on some behaviors and in some brain regions. There was considerable variability in the aged group in both their behavioral performance and their glucose utilization scores; thus, attempts were made to determine whether the variability in the degree of impairment within any particular behavioral test was correlated to the regional glucose utilization scores in any of the 45 brain regions analyzed. In two of the behavioral tests employed (i.e., one for learning and one for place navigation), the decline in performance correlated significantly with the decrement in regional glucose utilization. Moreover, the performance in these two tests showed significant correlation with glucose use in only five regions (dentate gyrus, medial septum-diagonal band area, hippocampal CA1, hippocampal CA3, and prefrontal cortex). These results show that the learning impairments in the aged rats are related to the extent of decrease in glucose utilization in restricted areas of the limbic system. In addition, the results show that the individual rats within an aged rat population develop cognitive impairments to a variable degree and that the aged rats with the most pronounced learning impairments are the ones exhibiting the most severe functional decrements, in terms of glucose utilization, in the septohippocampal system and the prefrontal cortex. This suggests that aging rats may be interesting not only for the study of the normal aging process, but also as a model of dementia.
\end{abstract}

Brain aging in humans is known to be a heterogeneous process. This heterogeneity is exemplified in the variation between individuals, the brain structures involved, and the nature and extent of demonstrable neurochemical and neuropathological changes. In particular, measurements of glucose utilization or oxygen consumption have made it possible to monitor changes in cerebral metabolic rate in diverse brain structures, related to the degree and severity of clinical symptoms in members of aged populations. Although aging in humans has generally been associated with a reduction in overall cerebral metabolism (Kety, 1956; Sokoloff, 1966; Kuhl et al., 1982), more recent studies using positron emission tomography indicate that a severe decrease in metabolic rate is seen only in subjects with pronounced cognitive impairments, while the

\footnotetext{
${ }^{1}$ We wish to thank Dr. Niels Diemer, Department of Neuropathology, University of Copenhagen, Denmark, for generously providing the TAS-Plus equipment. The skillful technical assistance of Carine Jönsson and Yvette Jönsson is gratefully acknowledged. We also thank Jan Berglund for drawing Figure 1. This work was supported by grants from the National Institute of Aging (AG 03766) and from the Swedish Medical Research Council (04X-3874).

${ }^{2}$ To whom correspondence should be addressed, at Department of Histology, Biskopsgatan 5, University of Lund, S-223 62 Lund, Sweden.

${ }^{3}$ Present address: Wellcome Surgical Institute, Glasgow University, Glasgow, Scotland, United Kingdom.
}

decline in normal, healthy, and nondemented aged humans is only slight (Benson, 1983; deLeon et al., 1983; Frackowiak and Gibbs, 1983; Rapoport, 1983). In fact, available data suggest that the severity of cognitive impairments is correlated with the magnitude of reduction in the cerebral metabolic rate and that this effect may be differentially expressed in different regions of the brain (Benson, 1983; deLeon et al., 1983; Frackowiak and Gibbs, 1983).

Age-related changes in cerebral metabolic rate have also been reported in rats, using Sokoloff's autoradiographic 2-deoxyglucose method (Smith et al., 1980; London et al., 1981; Smith and Sokoloff, 1982; Rapoport et al., 1983). These previous studies measured age-dependent metabolic changes in an unselected population of aged rats. It remains unclear whether cerebral metabolic rate in such unselected aged rat populations demonstrates any decline as a simple function of age (see Takei et al., 1983, and Rapoport, 1983, for discussion). In view of the heterogeneous changes reported in the aged human population, we have in the present study focused on the extent to which behavioral impairments in the aged rat population may be reflected in functional changes in specific brain structures, as assessed by the 2-deoxyglucose method. The results provide evidence that the individual animals within an aged rat population develop behavioral impairments to a variable degree and that, in selected limbic regions, including the septohippocampal system and the prefrontal cortex, the decrease in glucose use is well correlated with the severity of spatial learning impairment. 


\section{Materials and Methods}

\section{Subjects}

Female Sprague-Dawley rats (ALAB, Stockholm, Sweden) were used in this study. The rats were housed in standard laboratory cages in groups of three to six animals, with ad libitum access to food and water, and were maintained on a 12:12-hr light-dark cycle. Aged rats were bought as retired breeders, 9 to 11 months old. These aged rats were kept in the Lund University Hospital animal care facilities in a clean, controlled environment for an additional year before being transferred to the Department of Histology where the experiments were conducted. The young control rats were brought directly from the breeder to the Department of Histology at 2 months of age. All rats were allowed a further 3 to 4 weeks to stabilize before the experiments began. A total of 17 aged and 7 young control rats was tested.

\section{Behavioral tests}

The behavioral tests employed were similar to those described in our previous study (Gage et al., 1984b). In this previous set of experiments the tests employed were found to be sufficiently sensitive to measure significant changes in our population of aged rats between 21 and 24 months of age.

Water maze. The water maze test was adapted directly from Morris (1981). The test apparatus, illustrated in Figure 1, comprised a large circular pool made of PVC plastic ( $140 \mathrm{~cm}$ wide $\times 45 \mathrm{~cm}$ high) and was filled with water to a depth of $30 \mathrm{~cm}$. The water was made opaque by the addition of approximately 0.5 liter of dried milk powder and was thermostatically maintained at $26 \pm 1^{\circ} \mathrm{C}$. A transparent escape platform $\left(11 \times 11 \mathrm{~cm}^{2}, 29 \mathrm{~cm}\right.$ high) was placed in a fixed location in the tank, $1 \mathrm{~cm}$ below the water surface. The platform was not visible from just above water level (at least to the experimenter's eye), and transfer trials have indicated that escape onto the platform is not achieved by visual or other proximal cues (Morris, 1981). Many extra maze cues surrounded the maze and were available for the rats to use in locating the escape platform.

Each trial involved placing the rats into the water close to and facing the wall of the pool in one of four equally spaced locations ( 1 to 1 in Fig. 1). The rats were allowed to swim freely until they found the platform, onto which they would promptly climb to escape from the water. If a rat failed to locate the platform within $120 \mathrm{sec}$, it was then placed there by the experimenter. The intertrial interval was $60 \mathrm{sec}$, during which the rat remained on the platform. Each rat received 8 trials/day for 5 days. On the first 36 trials the platform remained in a constant location in the center of one quadrant equidistant from the center and the edge of the pool. For the final 4 trials the platform was removed from the pool in order to determine whether the animals had learned the task by using spatial or proximal cues.

On each of the first 36 trials two measures were recorded: (1) the latency to escape onto the platform, measured by a stopwatch; and (2) the length of the swim path, measured by observation and tracing the swim path onto a Hipad analogue-digital converter attached to an $\mathrm{ABC}$ 80 microprocessor. Computer analysis of the swim path also provided the distance swum in each of four quadrants in the pool (designated so that the cscape platform lay in the center of quadrant 4 on trials 1 to 36).

On the last trial (trial 37) the platform was removed from the pool and the percentage distance swum in each of the four quadrants was calculated over one 120 -sec period. In addition, the number of times the rat crossed the location where the platform had been previously located was recorded. 'These two measures enabled a determination of

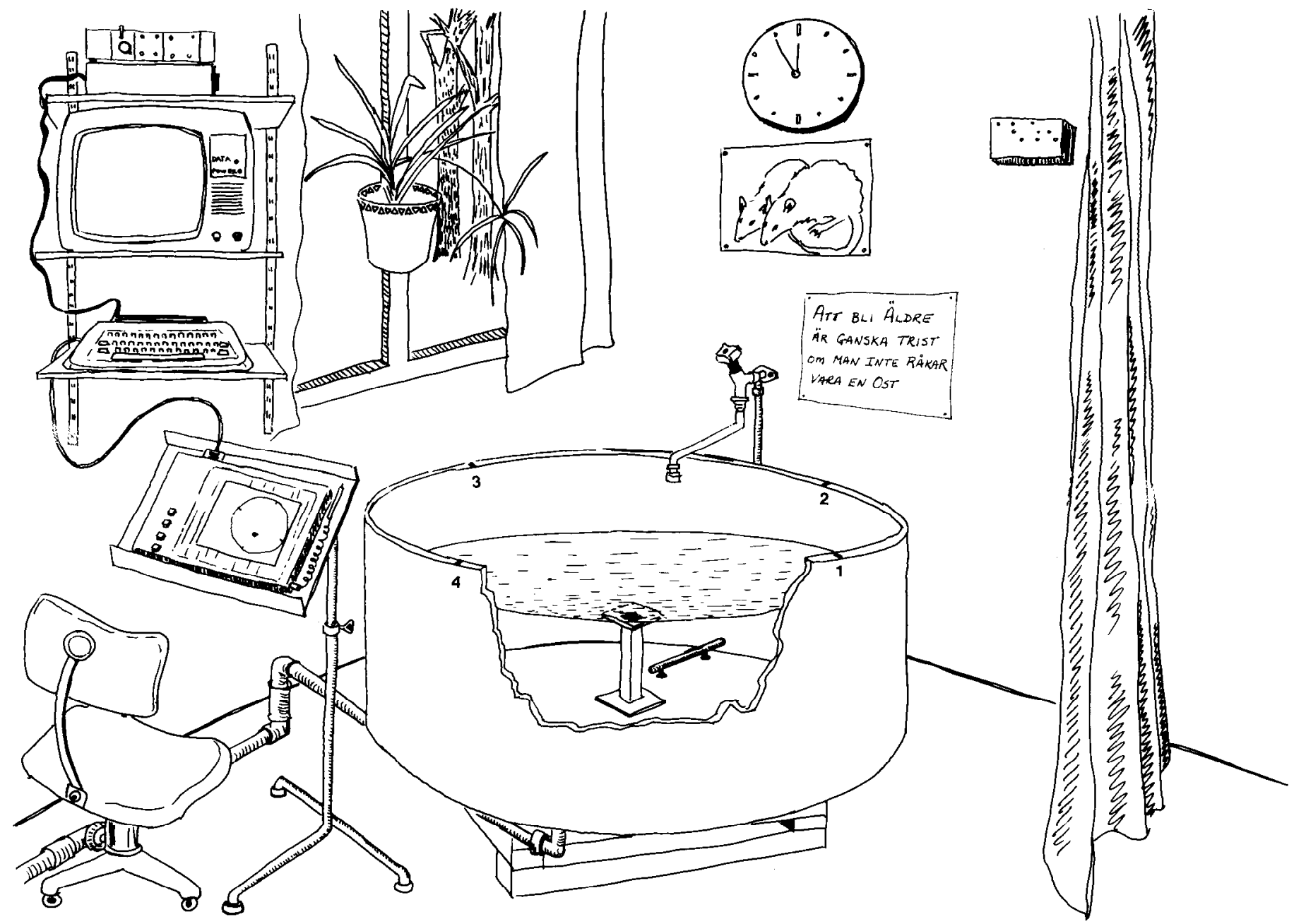

Figure 1. Schematic drawing of the water maze showing local cues around the pool, computer-coupled Hipad for tracing swim distance, and location of the invisible platform submerged in opaque water. Numbers 1 to 4 denote the four starting positions. 
whether the animals had learned the task using spatial or proximal cues.

Motor coordination test. The measures of motor coordination were adapted from the tests of Wallace et al. (1980), as follows (i) Square bridge: A 60-cm-long wooden bridge of $2 \times 2 \mathrm{~cm}$ cross-section was suspended between two safety platforms $60 \mathrm{~cm}$ above the bench surface. The rat was placed in the middle of the bridge, and the latency to reach the safety platform or to fall was recorded. (ii) Round bridge: 'This test was identical to the first test except that the bridge was made of a wood dowel rod of $2 \mathrm{~cm}$ diameter circular cross-section. (iii) Wire suspension: A taut, plastic-coated wire was stretched horizontally 60 $\mathrm{cm}$ above the bench surface. Rats grasped this wire with their forepaws and hung suspended or tried to climb up. The latency to fall was recorded.

In all tests, a pad of foam rubber was placed beneath the bridge or wire to cushion the rats' falls. On each test a maximum latency of 120 sec was allowed for the animal to fall. Any rat which took longer, or reached one of the safety platforms of the bridges, was attributed the maximum score $(120 \mathrm{sec})$. Each rat was tested on each of the tests three times in cyclical order.

Activity and exploration in an open field. Rats were tested in automated open field boxes $(50 \times 50 \times 60 \mathrm{~cm})$ made of gray plastic. In each box, four evenly spaced photocell beams perpendicular to each wall and $2 \mathrm{~cm}$ above the floor divided the box into 25 squares, $10 \times 10 \mathrm{~cm}$ each. Each photocell beam interruption was automatically registered as an activity count by on-line connection to an $\mathrm{ABC} 80$ microprocessor. The floor was raised $5 \mathrm{~cm}$ from the base of the box and contained 16 evenly spaced 2 -cm-diameter holes in a $4 \times 4$ array. An additional row of four photocell beams lay $1 \mathrm{~cm}$ below the holes and automatically recorded each exploratory nose poke. Each animal was tested in the dark for 1 hr.

Somatosensory reactivity. A startle box apparatus was used similar to that previously described to measure the unconditioned startle response to somatosensory stimulation (Turner and Gage, 1982). This type of test has been useful in the past to detect differences between young and aged animals (Campbell et al., 1980). The apparatus allows for the measurement of: (i) the latency to respond to stimulus (with threshold established at a preset level); (ii) the integral of the response over the time the initial response was above threshold; and (iii) the maximal amplitude of the response, which is the peak height of the integrated response. The startle was induced by a $0.5-\mathrm{mA}, 100-\mathrm{msec}$ scrambled electrical pulse given through the brass rods of the grid floor of the box. The mean response on each measure over five successive trials was taken as the animal's reaction. Previous experience with this stimulus level has demonstrated that the magnitude of the response allows for both increases and decreases in somatosensory reaction and that there is no apparent habituation of the response over 15 presentations.

Local cerebral glucose utilization. The autoradiographic $\left[{ }^{14} \mathrm{C}\right]-2$ deoxy-D-glucose method was performed on restrained but awake animals essentially according to the method of Sokoloff et al. (1977). Catheters were introduced into one femoral artery (to allow the intermittent monitoring of mean arterial pressure and the sampling of arterial blood) and into one femoral vein (for administration of the radioisotopic tracer) during a brief period $(\sim 20 \mathrm{~min})$ of light halothane (1\%) anesthesia. The animals were restrained by means of a loosefitting plaster cast around the pelvis and lower abdomen. The anesthetic gas mixture was discontinued and at least $2 \mathrm{hr}$ were allowed to elapse before any further manipulations were performed.

Briefly, the measurement procedure was initiated with an intravenous injection of $\left[{ }^{14} \mathrm{C}\right]-2$-deoxy-D-glucose $(125 \mu \mathrm{Ci} / \mathrm{kg}$; New England Nuclear) in $0.5 \mathrm{ml}$ of saline delivered over $30 \mathrm{sec}$. During the subsequent $45 \mathrm{~min}, 14$ timed arterial blood samples were withdrawn; from these, plasma concentrations of glucose and ${ }^{14} \mathrm{C}$ were determined by a semiautomated glucose oxidase assay (Beckman) and liquid scintillation analysis, respectively. At the end of the 45-min sampling period, the rats were killed by decapitation, and the brains were rapidly dissected out intact and frozen in precooled isopentane $\left(-45^{\circ} \mathrm{C}\right)$. The brains were sectioned in the coronal plane at $20 \mu \mathrm{m}$ thickness in a cryostat at $-22^{\circ} \mathrm{C}$. Autoradiograms were prepared by applying the dried sections to Kodak SB-5 $\mathrm{x}$-ray film together with a set of nine precalibrated ${ }^{14} \mathrm{C}$ containing plastic standards (concentration range, 44 to $1175 \mathrm{nCi} / \mathrm{gm}$ ) in a light-tight cassette for 7 days.

Tissue ${ }^{14} \mathrm{C}$ concentrations were measured densitometrically, using a Leitz TAS-Plus image-analyzing system, by reference to the precali- brated ${ }^{14} \mathrm{C}$ standards. For each anatomical region, bilateral determinations of optical density were made on six different autoradiographic images containing the area. ${ }^{14} \mathrm{C}$ tissue concentrations were calculated from the mean of those 12 readings.

Local cerebral glucose utilization was calculated using the operational equation for the technique (Sokoloff et al., 1977) from the ${ }^{14} \mathrm{C}$ cerebral tissue concentrations and arterial plasma ${ }^{14} \mathrm{C}$ and glucose profiles. The kinetic rate constants and the "lumped" constant used in the calculation of local rates of glucose utilization were the mean values reported previously for normal adult rats (Sokoloff et al., 1977).

\section{Autopsy}

All animals were screened at autopsy for gross pathological changes in their integument, viscera, and pituitaries. These observations, together with their body weights and general appearance prior to the experiment, were used to assess the general health status of each animal.

\section{Statistical analysis}

Comparisons between the aged and young rats on each separate behavioral test and each brain area measured for glucose utilization were carried out by calculating the Student's $t$ statistic. All $t$ statistics and corresponding $p$ values are presented in Tables I to V. Pearson product moment correlation coefficients were calculated between behavioral tests and between behavioral tests and glucose utilization in those brain regions which showed differences between young and aged rats with $t$ values of at least $p<0.05$.

\section{Results}

During the course of the experiment, 6 of the 17 aged rats died and, thus, they were excluded from the final analysis. Of the remaining 11 aged animals 3 had body tumors, 5 had slight audible respiratory abnormalities, and 2 had visible pituitary swelling; 5 animals had none of these pathological signs. No significant or meaningful relationship was observed between the physical signs of aging in these rats and their performance on any behavioral task that we measured. In other words, there were old animals with tumors or respiratory problems which performed as well as young animals, just as there were aged animals which showed severe behavioral impairments but had no visible tumors or respiratory difficulties. In addition there was no significant correlation between these physical signs of aging and rates of glucose utilization.

\section{Behavior}

Consistent with previous results (Marshall and Berrios, 1979; Wallace et al., 1980; Gage et al., 1984a, b), the 22- to 24-monthold aged rats were significantly impaired on several behavioral measures when compared with the 3 -month-old young controls. In Table I, selected measures from each of the four different behavioral tests (i.e., water maze (WM), motor coordination test (MC), open field test (OF), and somatosensory reactivity test (SR)) are listed for the young and aged rats as groups. The various measures have been ordered, from top to bottom, according to the magnitude of the difference measured between the aged and young groups.

Water maze. The aged rats were significantly impaired in the water maze test on five different measures: (1) the distance swum in the fourth quadrant (i.e., the quadrant of the platform) in the first trial after the platform had been removed (Q4); (2) the number of times the previous location of the removed platform was crossed in the first trial after the platform had been removed (platform crossings); (3) and (4) the distances swum in the second and third quadrants (i.e., non-platformcontaining quadrants) in the first trial after the platform had been removed; and (5) the mean latency in seconds for the rats to find the invisible platform in the last four trials prior to platform removal (escape latency). These results show that the aged group of rats was impaired in the acquisition of spatial 
TABLE I

Differences between aged (22- to 24-month-old) and young (3-monthold) rats in 16 different behavioral measures, ranked according to their $t$-values obtained by two-tailed Student's t test

\begin{tabular}{|c|c|c|c|c|c|}
\hline & Behavior & $\begin{array}{l}\text { Young } \\
(n=7)\end{array}$ & $\begin{array}{c}\text { Aged } \\
(n=11)\end{array}$ & $t$ & $p$ \\
\hline \multicolumn{6}{|c|}{ mean $\pm S E M$} \\
\hline WM: & $\begin{array}{l}{ }^{a} \text { Swim distance in } \\
\text { Q4 }(\%)^{b}\end{array}$ & $66 \pm 3$ & $30 \pm 5$ & -5.30 & $<0.001$ \\
\hline WM: & Platform crossings & $11 \pm 1$ & $3 \pm 1$ & -5.10 & $<0.001$ \\
\hline MC: & $\begin{array}{l}\text { Latency to fall, } \\
\text { round bridge (ser) }\end{array}$ & $90 \pm 12$ & $23 \pm 8$ & -4.84 & $<0.001$ \\
\hline SR: & $\begin{array}{l}\text { Maximum reac- } \\
\text { tion (units) }\end{array}$ & $19 \pm 2$ & $10 \pm 1$ & -3.78 & $<0.01$ \\
\hline OF: & $\begin{array}{l}\text { Exploration (nose } \\
\text { pokes/hr) }\end{array}$ & $42 \pm 10$ & $15 \pm 3$ & -3.18 & $<0.01$ \\
\hline SR: & $\begin{array}{l}\text { Reaction latency } \\
(\mathrm{msec})\end{array}$ & $29 \pm 2$ & $55 \pm 7$ & 2.89 & $<0.05$ \\
\hline WM: & $\begin{array}{l}\text { Swim distance in } \\
\text { Q3 }(\%)\end{array}$ & $11 \pm 2$ & $23 \pm 6$ & 2.81 & $<0.05$ \\
\hline WM: & $\begin{array}{l}\text { Swim distance in } \\
\text { Q2 }(\%)\end{array}$ & $5 \pm 1$ & $53 \pm 15$ & 2.53 & $<0.05$ \\
\hline WM: & $\begin{array}{l}\text { Escape latency } \\
\text { (sec) }\end{array}$ & $7 \pm 2$ & $53 \pm 15$ & 2.49 & $<0.05$ \\
\hline SR: & $\begin{array}{l}\text { Reaction integral } \\
\text { (units) }\end{array}$ & $6 \pm 1$ & $4 \pm 1$ & -2.05 & $>0.05$ \\
\hline MC: & $\begin{array}{l}\text { Latency to fall, } \\
\text { square bridge (sec) }\end{array}$ & $113 \pm 5$ & $96 \pm 9$ & 1.37 & $>0.05$ \\
\hline OF: & $\begin{array}{l}\text { Activity (beam } \\
\text { crossings/hr) }\end{array}$ & $1491 \pm 200$ & $1651 \pm 179$ & 0.58 & $>0.05$ \\
\hline WM: & $\begin{array}{l}\text { Swim distance in } \\
\text { Q1 }(\%)\end{array}$ & $21 \pm 2$ & $23 \pm 4$ & 0.39 & $>0.05$ \\
\hline MC: & $\begin{array}{l}\text { Latency to fall, } \\
\text { wire suspension } \\
(\mathrm{sec})\end{array}$ & $15 \pm 5$ & $16 \pm 1$ & 0.26 & $>0.05$ \\
\hline
\end{tabular}

${ }^{a}$ Abbreviations used are: WM, water maze test; MC, motor coordination test; OF, open field test; SR, somatosensory reactivity test.

${ }^{b}$ Measuring units for each test are in parentheses.

learning in the water maze task and was impaired, in comparison with the young controls, in its ability to use spatial cues in the test (see Table II and Fig. 2).

Motor coordination. The aged rats were impaired in their ability to maintain balance on the round bridge, where they showed a marked reduction in the latency to fall off. The mean latencies to fall off from the square bridge and from the taut wire (which is primarily a measure of motor strength) were not significantly less than those of the controls.

Open field. Although the spontaneous activity of the aged rats in the open field was similar to that of the young controls, their activity was significantly impaired on the exploratory measure of the test; i.e., the number of nose pokes in the holes of the floor.

Somatosensory reactivity. In the startle response to an electric foot shock, the maximum amplitude of the response was significantly reduced, and the latency to react was significantly prolonged in the aged group, whereas the difference in the total integrated response (reaction integral) did not reach significance.

\section{Correlation between different behavioral measures}

These results show that the aged rats as a group were significantly impaired on a number of cognitive and noncognitive behavioral tests. However, consistent with our previous observations (Gage et al., 1984b), the degree of impairment in each test, as well as the patterns of deficits on the different tests, varied widely among the individual rats within the aged group. This is exemplified for the water maze test in Figure 2, where some of the aged rats performed similarly to the young control rats (Fig. 2, bottom), whereas others were completely unable to solve the task (Fig. 2, middle). The extent to which the different behavioral measures covaried in the individual aged rats was therefore analyzed statistically by multiple correlations.

In Table III, 10 of the behavioral measures in Table I have been examined statistically for their intercorrelation in the aged group of rats. Three of these measures (behaviors 1 to 3 ) reflect the spatial learning ability in the water maze task; two measures (behaviors 4 and 5) reflect the motor coordination abilities on the round and square bridge test; three measures (behaviors 6 to 8 ) reflect the somatosensory reactivity in the startle response test; and two measures (behaviors 9 and 10) give the general locomotor activity and exploratory response in the open field test. Of the 45 different possible correlations analyzed only three reached significance at the $p<0.01$ level and three reached significance at the 0.05 level. Thus, several of these correlations could be attributed to chance. Nevertheless, it is interesting to note that several measures of motor coordination, somatosensory reactivity, and general open field activity showed significant correlations with one another (i.e., 5 versus 7,6 versus 9 , and 7 versus 8 ), whereas the different measures of cognitive function were positively correlated only with one another ( 1 versus $2, r=0.838$ ). Thus, escape latency in the last four trials prior to platform removal (behavior 1) (which is a measure of acquisition of the learned performance in the water maze task) was correlated with the number of platform crossings in the first trial after platform removal (behavior 2) (which is a measure of the use of spatial cues in the task). There was a significant negative correlation between platform crossings and latency to fall from the square bridge (but not in the comparable test on the round bridge); this correlation does not seem meaningful to us.

The intercorrelation analyses thus indicate that the impairments in cognitive function of the aged rats were generally independent of the age-related impairments in other behavioral tasks, which leads us to conclude that the aged rats constituted a behaviorally heterogeneous population.

\section{Local cerebral glucose utilization}

Local rates of glucose utilization were determined in 45 anatomically discrete and functionally diverse cerebral structures, as summarized in Table IV. The results are presented in hierarchical order, from the structures which display the most significant differences between aged and young groups $(p<$ $0.001)$ to those in which no significant differences were apparent $(p>0.05)$.

Of the 45 structures analyzed, 16 showed some degree of significant quantitative decrease in local glucose utilization ( $p$ $<0.05$ ), the difference in mean values being on the order of 10 to $20 \%$. In keeping with previously reported data (Smith et al., 1980) functional activity was decreased in the aged group as a whole in the primary visual cortex and the superior colliculus, as well as in caudate-putamen and the dentate gyrus. In addition, the aged rat group in the present experiments also displayed significant decreases (of at least $p<0.05$ ) in a variety of other structures. Of particular relevance to the observed learning impairments in the water maze task were the decreases in glucose utilization seen in the components of the limbic system and closely associated regions. Thus, changes were observed in the ventral septal band area, the hippocampal CA1 and $\mathrm{CA}$ regions, the anterior cingulate cortex, the anteromedial frontal cortex (i.e., the prefrontal cortex), the mediodorsal thalamic nucleus, and the medial and dorsal raphe nuclei. 


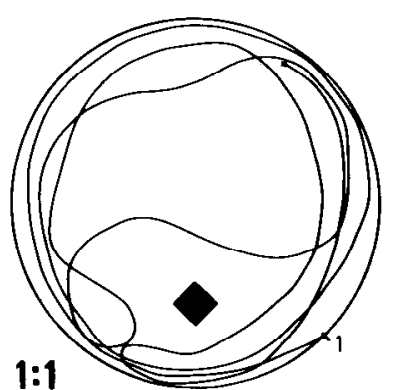

Escape latency 120

$\%$ in $4^{\text {th }}$ quad: -

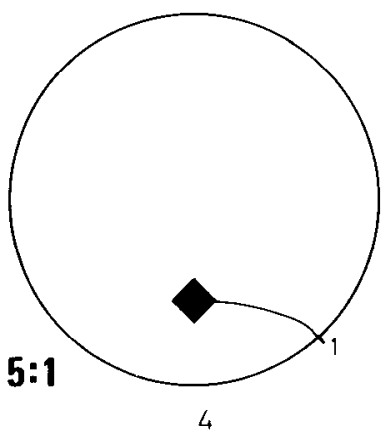

$-$
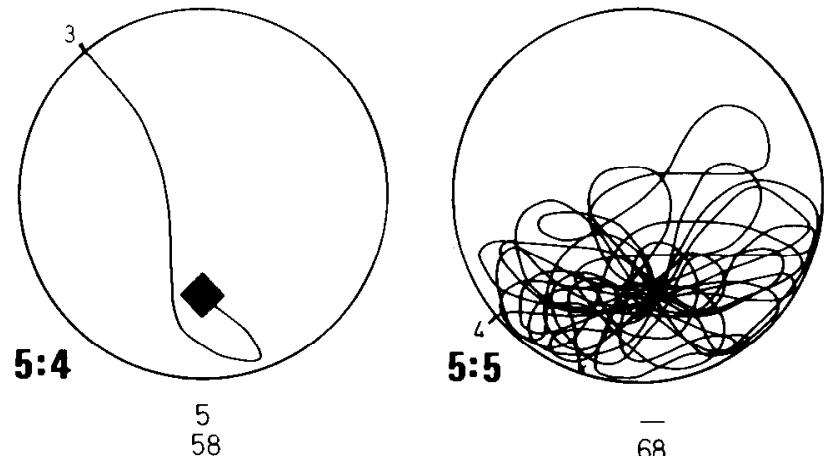

68

AGED

IMPAIRED (I2)

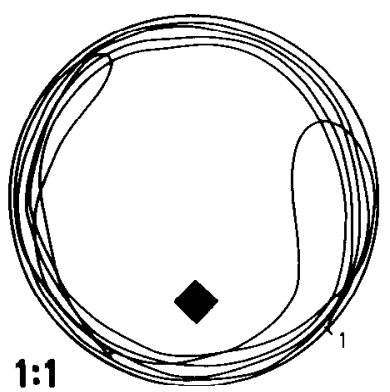

Escape latency 120

$\%$ in $4^{\text {th }}$ quad: -

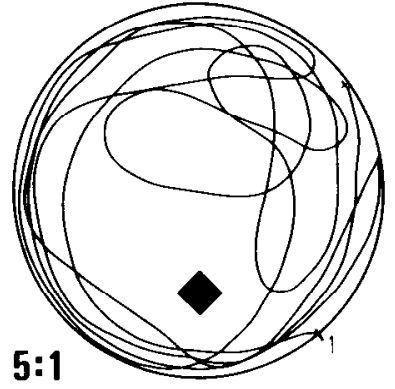

120

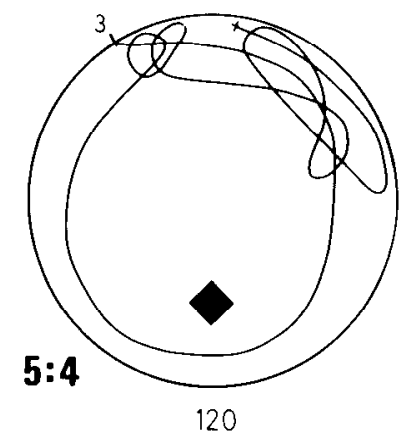

10

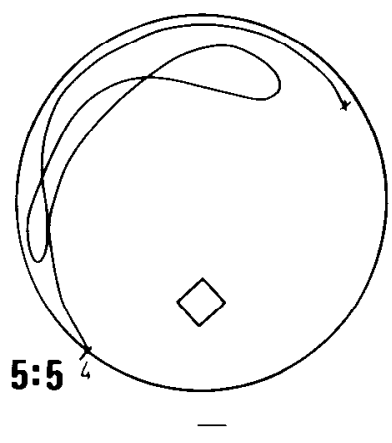

0

\section{AGED}

NON-IMPAIRED (I I)

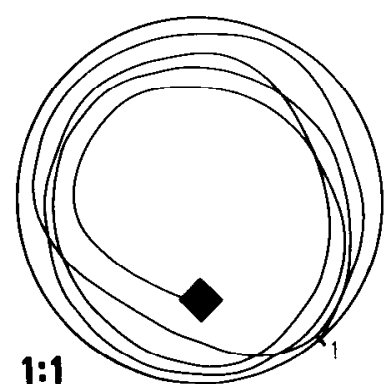

1:1

Escape latency 38

$\%$ in $4^{\text {th }}$ quad: -

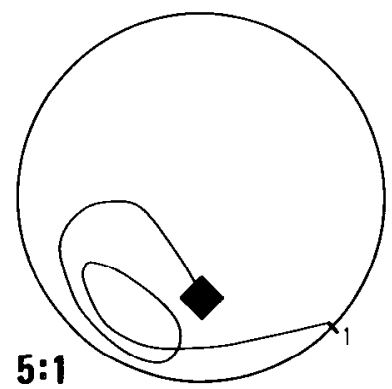

11

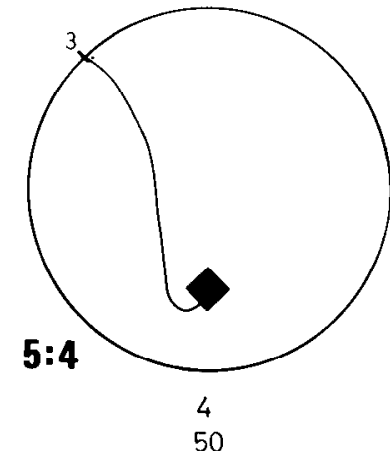

50

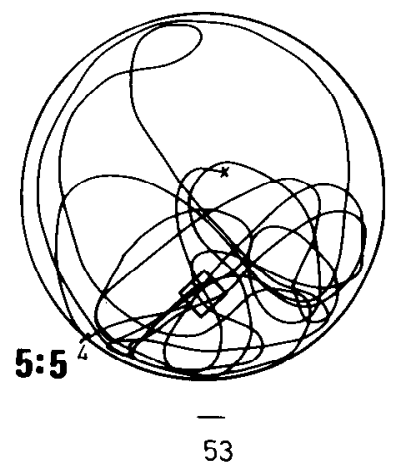

Figure 2. Direct tracings of water maze performance of a young control rat (YNC 2), an aged impaired rat (I2), and an aged nonimpaired rat (II 1), on four different trials during the week of testing; 1:1 corresponds to the first trial on day 1, 5:1 corresponds to the first trial on day $5,5: 4$ corresponds to fourth trial on day 5, and 5:5 corresponds to the fifth trial on the fifth day, when the platform had been removed from the pool. The solid square marks the location of the platform in the pool. The open square marks the location of where the platform had been on the previous trials. Escape latency corresponds to the time required to find the platform on each trial; \% in 4 th quad is the percentage of the total distance swum in the trial prior to platform removal (5:4) or in the trial following platform removal (5:5). The smaller numbers next to the pools $(1,3$, and 4 ) correspond to the starting location (as indicated in Fig. 1) for the rat on those trials.

TABLE II

Regional glucose utilization of the three rats illustrated in Figure 2

\begin{tabular}{lcccccccccccccccc}
\hline Rat $^{\alpha}$ & DG $^{b}$ & MS-DB & DR & CA1 & HB & CA3 & VCX & MDT & VLT & SNPR & PFC & MR & ACCx & SC & CP & CN \\
\hline Young control (YNC 2) & $56^{c}$ & 52 & 70 & 64 & 60 & 54 & 73 & 80 & 64 & 44 & 92 & 76 & 76 & 68 & 70 & 69 \\
Aged impaired (I 2) & 35 & 40 & 58 & 47 & 47 & 37 & 77 & 68 & 54 & 38 & 70 & 55 & 64 & $\mathbf{6 8}$ & $\mathbf{6 8}$ & 65 \\
Aged nonimpaired (II 1) & 52 & 52 & 58 & 63 & 56 & 51 & 56 & 77 & 52 & 32 & 89 & 61 & 80 & 57 & 53 & 58 \\
\hline
\end{tabular}

${ }^{a}$ These rats are indicated, respectively, as $a, b$, and $c$ in Figure 3.

${ }^{b}$ Abbreviations used are: DG, dentate gyrus; MS-DB, medial septum-diagonal band area; DR, dorsal raphe nucleus; CA1, hippocampus CA1 molecular layer; HB, medial habenula; CA3, hippocampus CA3 area; VCX, visual cortex; MDT, mediodorsal thalamus; VLT, ventrolateral thalamus; SNPR, substantia nigra pars reticulata; PFC, prefrontal cortex; MR, medial raphe nucleus; ACCX, anterior cingulate cortex; SC, superior colliculus; $\mathrm{CP}$, caudate-putamen; $\mathrm{CN}$, cerebellar nuclei.

${ }^{c}$ Values are micromoles per $100 \mathrm{gm}$ per minute. 
TABLE III

Intercorrelations of performances in different behavioral tests in the aged group of rats The correlation coefficients $(r)$ are those obtained in the Pearson product moment correlation test.

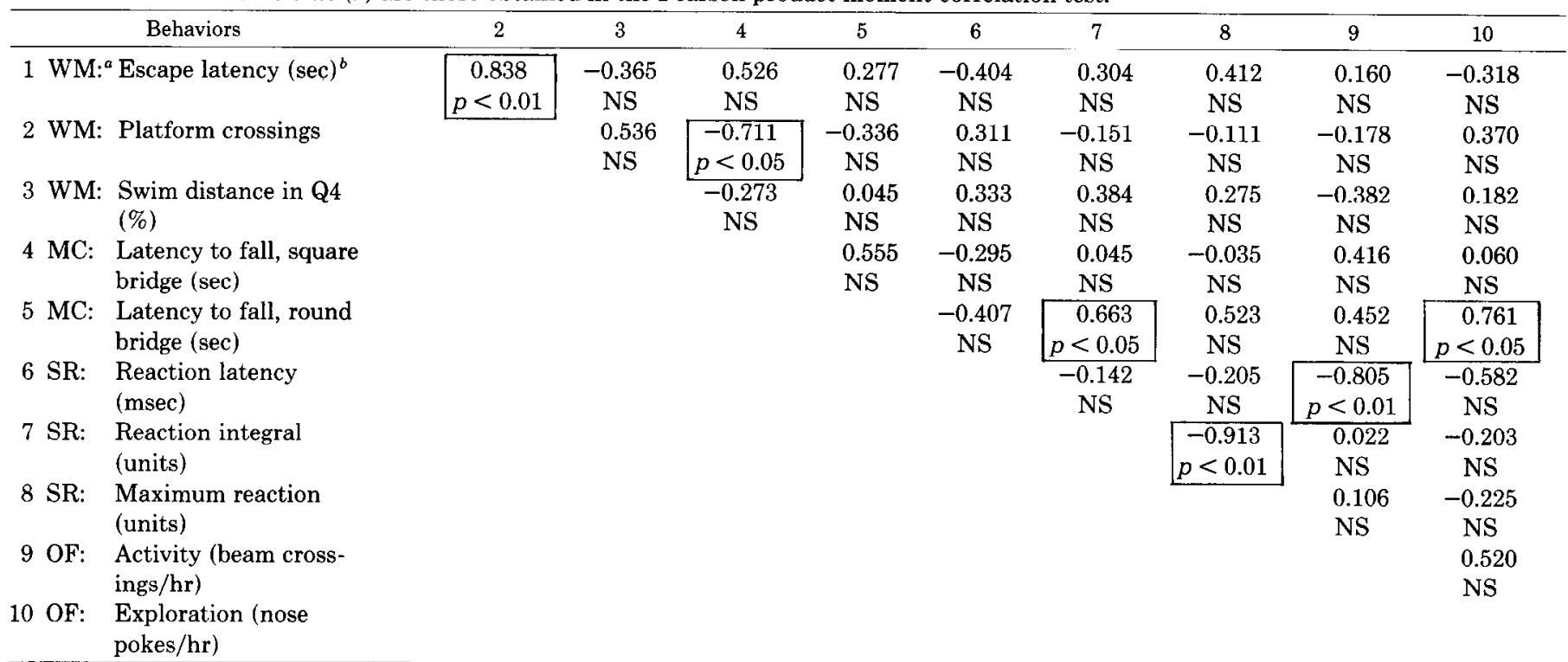

${ }^{a}$ Abbreviations used are: WM, water maze test; MC, motor coordination test; OF, open field test; SR, somatosensory reactivity test; NS, not significant.

${ }^{b}$ Measuring units for each test are in parentheses.

Although these areas displayed significant decreases in glucose use in the aged group as a whole, when compared to young controls, there was considerable variability in the magnitude of the decreases observed within the aged group (see Table IV). As was the case in the behavioral measures, regional rates of glucose use in the aged group ranged from near normal (but never greater than young controls) to large decreases from control levels. In contrast, the variance of measured rates of glucose use in young rats was small (see Table IV).

\section{Correlations of behavioral performance in aged rats and rates of local glucose use}

In order to assess whether the behavioral impairments in the aged rat group were related to anatomically differential decreases in local glucose utilization, the local glucose use in those 16 regions of the brain which showed decreases reaching at least $p<0.05$ (see Table IV) were correlated with the nine behavioral measures which exhibited decreases reaching at least $p<0.05$ (see Table I).

Only two of the behavioral measures (escape latency and platform crossings in the water maze test) were significantly correlated with glucose use in any of the brain regions analyzed. Moreover, these two behaviors showed significant correlation (at the $p<0.01$ level) with only 5 of the 16 brain regions included in the analysis (Table V): the three areas of the hippocampal formation measured (i.e., dentate gyrus, and the hippocampal CA1 and CA3 fields), the medial septum-diagonal band area, and the prefrontal cortex. The scatter plots in Figure 3 give the glucose use values for the individual aged rats (Fig. 3 , solid circles) and young controls (Fig. 3, open circles) plotted against their acquisition in their water maze task (escape latency) and their ability to use spatial cues in the localization of the platform (platform crossings). Two of the structures illustrated in Figure 3 (prefrontal cortex and dentate gyrus) represent regions where the glucose use and behavioral measures were highly correlated, and the other two (visual cortex and cerebellar nuclei) illustrate regions where the glucose use values were significantly decreased in the aged group (Table IV) but not correlated with any behavioral measure.
Escape latency, which is a measure of acquisition in the water maze task, was negatively correlated with local glucose use in all five regions, indicating that the rats which took the longest time to find the platform in the last four trials prior to platform removal had the lowest glucose utilization values (top left panels in Fig. 3). Platform crossings, on the other hand, which measure the use of spatial cues in the task, were positively correlated with local glucose use only in dentate gyrus and prefrontal cortex, indicative that the rats which were most impaired in the use of spatial cues in the task had the lowest glucose uptake values in these two regions (bottom left panels in Fig. 3).

Figure 2 illustrates the behavioral performance of a young rat (Fig. 2, top), an aged rat which exhibited severe behavioral impairments on the water maze task (Fig. 2, middle), and an aged rat with water maze performance similar to that of the young control group (Fig. 2, bottom). Table II (below Fig. 2) presents the regional glucose utilization values for each of the three selected cases (marked $a, b$, and $c$ in Fig. 3), for those 16 brain regions which showed significant differences between the aged and young groups at least at the $p<0.05$ level. Although presentation of data from individual animals does not necessarily represent the entire sample, it can be seen that the individual values in this case match well with the correlations for the entire aged group presented in Table V.

\section{Discussion}

The present results show: $(i)$ that local glucose utilization was significantly reduced in selected brain regions in the aged rats as a group; ( $i i)$ that behavioral performance was significantly impaired in the aged rats as a group; (iii) that the deterioration in behavioral performance was not similar in all aged rats; (iv) that the behavioral impairments manifested themselves differently in different rats, such that the cognitive impairments did not necessarily correlate with impairments in sensorimotor or motor performance; and $(v)$ that the measures of cognitive impairment, as assessed in the spatial learning test, were highly correlated with the decrements in glucose utilization in areas of the septohippocampal system and prefrontal cortex. 
TABLE IV

Local cerebral glucose use

Analyses were made to compare regional rates of glucose use in 45 brain areas of aged (22- to 24 -month-old) and young (3-month-old) rats (two-tailed Student's $t$ test). Means \pm SD are expressed as micromoles per 100 gm per minute.

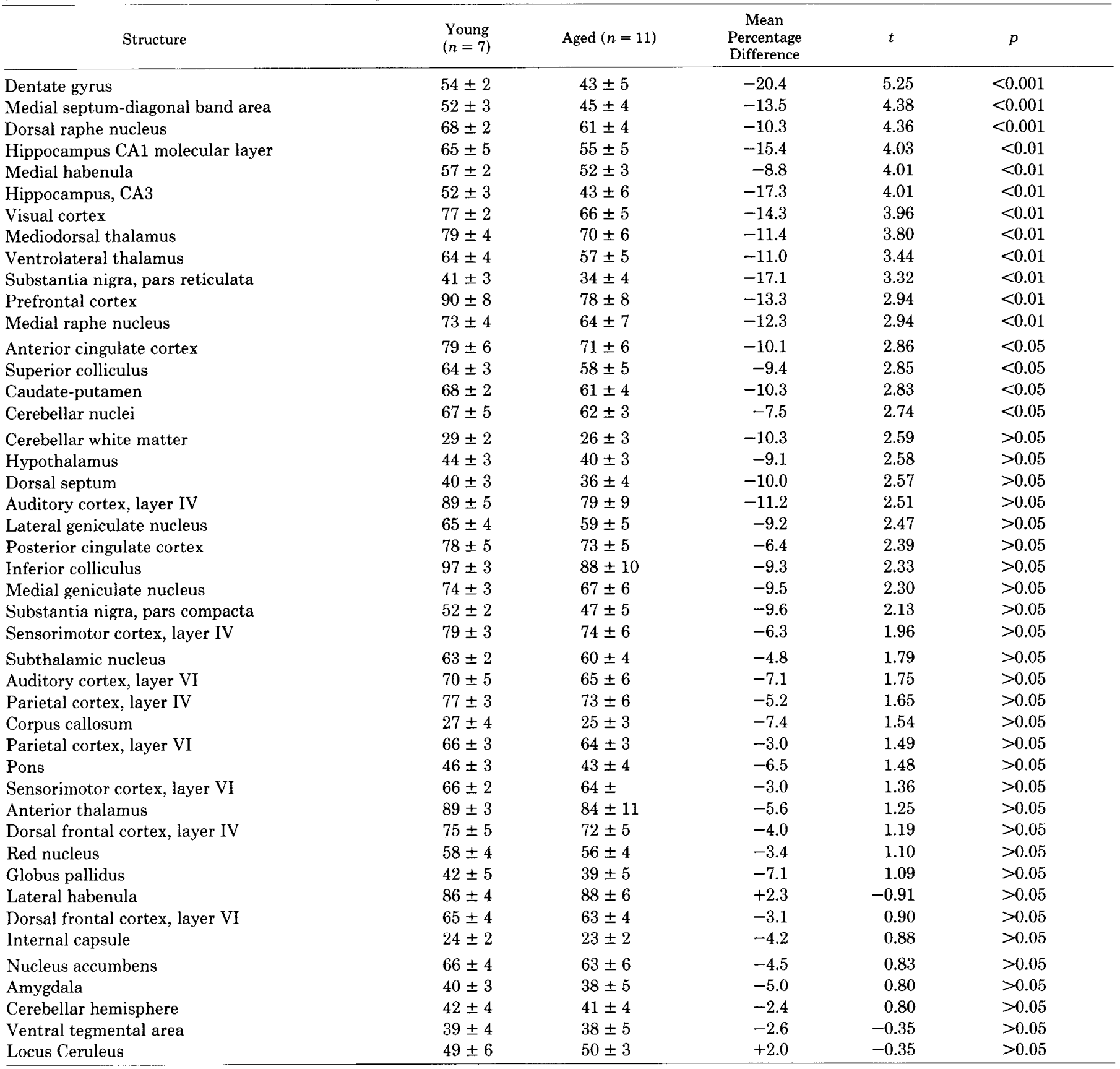

General metabolic activity of defined CNS regions, as assessed by the 2-deoxyglucose method, can reflect the functional state or physiological activity in major neuronal circuitries operating in these areas (see Sokoloff et al., 1977, and Sokoloff, 1981 for review). Of particular interest in the present context is the observation that regional glucose utilization can also change significantly in relation to changes in behavioral performance in young, awake animals (Matsunami et al., 1981; Schwartzman et al., 1981; Martinez et al., 1982; Matsunami and Kubota, 1983; Shimada et al., 1983). For this reason the 2deoxyglucose method should afford a valuable tool for the study of decrements in CNS function with age.

Some initial questions concerning the application of the 2deoxyglucose method in developmental and aging studies are required prior to a more specific discussion of the present results. Concern has recently been raised that the rate constants, and particularly the "lumped" constant, which were originally characterized for calculation of rates of glucose utilization in young adult rats (Sokoloff et al., 1977) may not be applicable in an aged population (Rapoport et al., 1983; Rapoport, 1983). Then, values calculated in aged rats using standard rate constants would underestimate the actual metabolic rates in the tissue. To date, however, altered values for the lumped constant have been reported only under conditions of severe hypo- and hyperglycemia. If conditions prevail in which plasma glucose levels fall below $40 \mathrm{mg} / 100 \mathrm{ml}$, the lumped constant rises dramatically (Suda et al., 1981). Conversely, in hyperglycemia, with plasma glucose concentration above 250 to $300 \mathrm{mg} /$ 
TABLE V

Correlations of behavioral performance in aged rats and rates of local cerebral glucose use

Nine behavioral measures (those in Table I, which showed difference at least at the $p<0.05$ level) were correlated with the Pearson product moment correlation test with the local glucose use in the 16 brain regions in Table IV showing significant $(p<0.05)$ reductions in the aged group as a whole. The two behaviors and the five regions listed were the only measures showing correlations significant at the $p<0.01$ level.

\begin{tabular}{lcc}
\hline \multirow{2}{*}{ Brain Region } & \multicolumn{2}{c}{ Behavioral Test } \\
\cline { 2 - 3 } & \multicolumn{1}{c}{$\begin{array}{c}\text { Escape } \\
\text { latency }\end{array}$} & $\begin{array}{c}\text { Platform } \\
\text { crossings }\end{array}$ \\
\hline Hippocampus, CA1 molecular layer & $\begin{array}{l}r=-0.749 \\
(p<0.01)\end{array}$ & $\mathrm{NS}^{a}$ \\
Dentate gyrus & $\begin{array}{l}r=-0.868 \\
(p<0.001)\end{array}$ & $r=+0.772$ \\
Hippocampus & $\begin{array}{l}(p<0.01) \\
(p<0.005)\end{array}$ & $\mathrm{NS}$ \\
Medial septum-diagonal band area & $\begin{array}{l}r=-0.804 \\
(p<0.005)\end{array}$ & $\mathrm{NS}$ \\
Prefrontal cortex & $r-0.887$ & $r=+0.778$ \\
& $(p<0.001)$ & $(p<0.005)$ \\
\hline
\end{tabular}

${ }^{a}$ NS, not significant.
$100 \mathrm{ml}$, there appears to be a moderate, progressive fall in the value of the lumped constant (Schuier et al., 1981). The evidence for age-related decrease in the lumped constant is, so far, somewhat inferential. It has been reported that the close relationship which exists between cerebral blood flow and glucose utilization is reset to different levels between 3 and 12 months of age such that, in effect, local rates of glucose use decrease per unit of blood flow. Sufficient data on these parameters so far are not available to allow for any definite conclusions on this issue, but the results reported by Rapoport et al. (1983) and Rapoport (1983) necessitate a cautious attitude, particularly toward the interpretations of differences between animals in different age groups.

The possibility that the decreased rates of glucose use observed in the normoglycemic aged rats in the present study could be due to an altered setting of the "lumped" constant cannot, therefore, be totally discounted. However, if the lumped constant was the only factor to change in this group, then quite large decreases (greater than $25 \%$ ) would be necessary to account for the significant decreases in the calculated rates of local glucose utilization. Moreover, the regional specificity of the changes in each aged animal and the heterogeneity of these alterations in glucose use within the aged group would require that the lumped constant should change differentially within specific brain structures, as well as changing at different stages
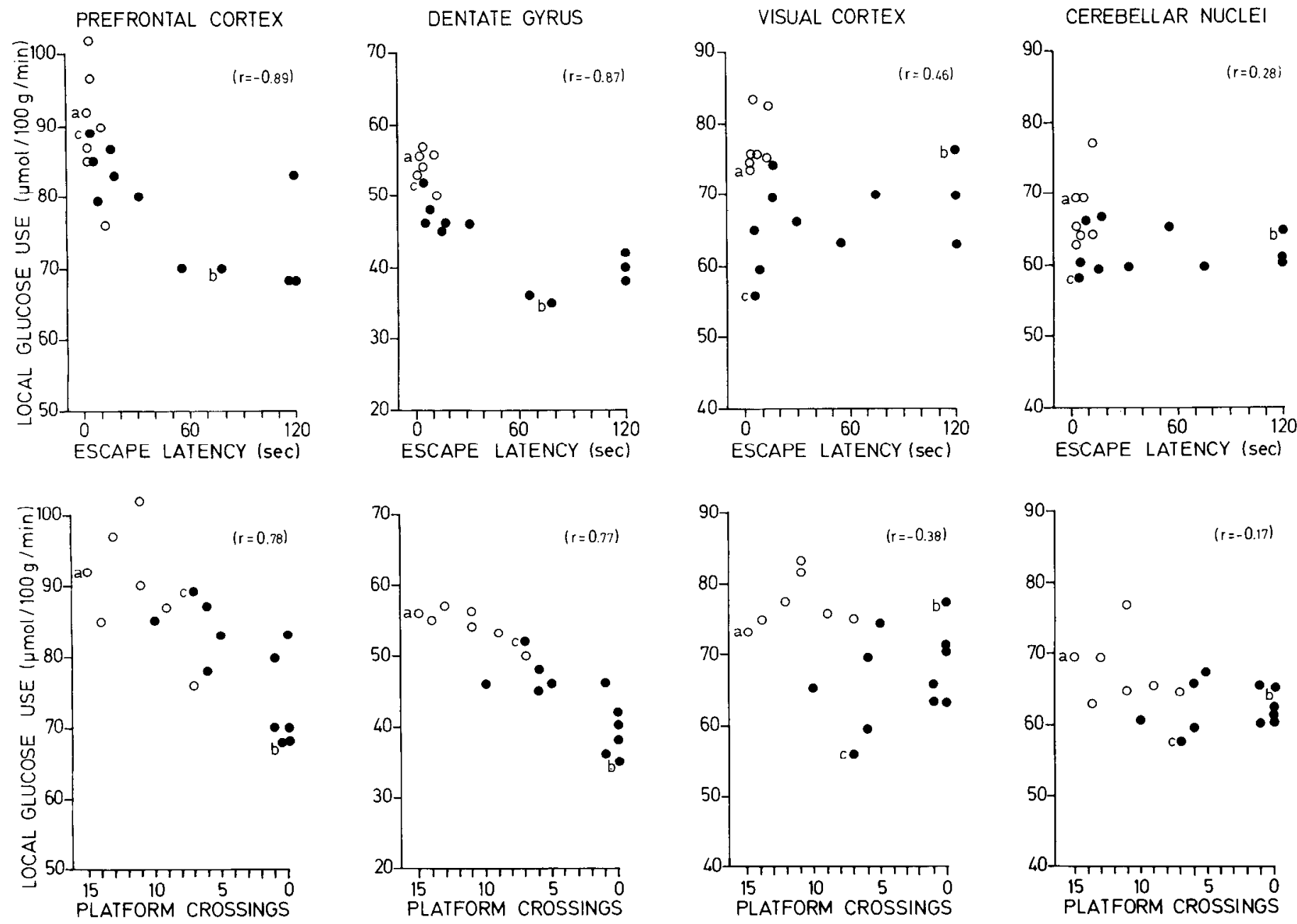

Figure 3. Scatterplots showing relationship between local glucose utilization and escape latency (top panels) and platform crossings (bottom panels). Four different brain regions are plotted against the two behavioral measures. The correlation coefficients in the upper right-hand corner of every graph correspond to the correlations obtained from the 11 aged animals only $(\circlearrowleft)$ : The seven young animals are represented by open circles (O) and are shown as reference points. Symbols $a, b$, and $c$ are to the left of three selected case animals depicted in Table II and Figure 2. 
of the aging process in individual animals. In view of the strong correlations which we have demonstrated between decreased cognitive function and measured rates of glucose use within a limited number of brain areas, it appears more likely that the decreases in glucose use directly reflect age-related alterations in the functional capacity of these brain regions. In general, it seems that the concerns over the lumped constant expressed by Rapoport et al. (1983) are of less relevance in comparisons in the same age group than in comparisons between individuals of different age groups.

Previous 2-deoxyglucose studies in aged rats using the ageinvariant "lumped" constant (Smith et al., 1980; London et al., 1981; Smith and Sokoloff, 1982; Rapoport et al., 1983) have reported significant age-dependent reductions in glucose use in several cortical areas, in caudate-putamen and globus pallidus, and in parts of the hippocampal formation. The present results generally confirm these findings. Thus, as a group, our 22- to 24-month-old rats exhibited significant decrements, on the order of 10 to $20 \%$, in 16 of the 45 structures analyzed. The regions with decreased glucose use included prefrontal, anterior cingulate, and visual cortex; caudate-putamen and pars reticulata of the substantia nigra; and the medial septum-diagonal band area, dentate gyrus, and hippocampal CA1 and CA3 fields. Moreover, several subcortical structures intimately associated with the limbic system, such as the mediodorsal thalamus and the dorsal and medial raphe nuclei, were also significantly decreased.

Consistent with the hypothesis that the decreases recorded in cerebral metabolic rate reflect functional changes, the aged rats as a group demonstrated significant impairments in several cognitive and noncognitive behavioral tasks. However, consistent with our previous study (Gage et al., 1984a, b), the pattern of behavioral deficits as well as the magnitude of the deficit on any single behavioral measure differed markedly between the different individuals in the group. Not all animals showed deficits: some rats were impaired only on some tasks, whereas others were impaired on some measures of all tasks. The intercorrelation analyses performed both on the present group of rats and on our previous group of rats of similar age (Gage et al., 1984a, b) suggest that different measures of cognitive impairment, derived from the water maze test, were highly correlated between the different aged rats but that the measures of cognitive impairment intercorrelated poorly with the measures of impairment in the motor and the somatosensory reactivity tests.

The results therefore suggest that cognitive and noncognitive impairments may develop with different time courses and, to a varying degree, in different individuals of an aging rat population. As a consequence, only a subpopulation of any group of aged rats will show significant cognitive impairments, and these rats will not necessarily be impaired on noncognitive tasks. In our strain of female Sprague-Dawley rats about one-third to one-fourth of the animals have demonstrated severe cognitive impairments in the water maze test at the age of 20 to 24 months. This subgroup, on the other hand, is at least as severely impaired in the water maze test as young rats subjected to bilateral fimbria-fornix lesions (Gage et al., 1982), bilateral hippocampal ablations (Morris et al., 1982), or bilateral prefrontal cortex damage (Kolb et al., 1983).

The significance of the observed decrements in regional glucose use in the aged rats gains particular support not only from the strong correlations that are seen between the cognitive behavioral measures in the water maze test and the reductions in glucose use, but also from the remarkable anatomical specificity of these intercorrelations. Thus, the measures of cognitive impairment were correlated with the decrement in glucose use in regions of the septohippocampal system and the prefrontal cortex (i.e., brain structures that are importantly implicated in learning and memory tasks), and it did not occur in any of the 11 other structures that exhibited significant reductions in glucose use in the aged rat group. Furthermore, none of the measures of motor or sensorimotor performance were correlated with any glucose use values in any of the 16 brain regions included in the correlation analysis.

The correlation between cognitive impairment and decrement in regional glucose use in the aged rats appears to be analogous to current findings in aged humans based on positron emission tomography. Thus, measurements of both oxygen consumption (Frackowiak and Gibbs 1983) and glucose utilization (Benson, 1983; Rapoport, 1983) in aged humans indicate that the cerebral metabolic rate is markedly depressed only in patients with symptoms of dementia and that the magnitude of the reduction in some regions is at least grossly correlated to the severity of the dementia symptoms. In humans, cognitive impairments have been reported to occur with normal "dementia-less" aging (Birren et al., 1963). However, there is substantial evidence that the development of dementia is correlated with specific neuropathological processes that are either different from or at least much more extensive than in normal aging (Ingvar et al., 1977; Perry et al., 1978; Davis et al., 1980; Whitehouse et al., 1982). On the basis of the present findings we would like to propose that a similar conclusion also applies in rats: the development of cognitive impairments is a relatively specific process in the aging population which can be correlated with measurable functional decrements in specific limbic forebrain regions. Indeed, since these functional decrements and their behavioral correlates share many features with the appearance of dementia in aging humans, they could serve to define a "dementia-like" subpopulation of aging rats. This suggests that aging rats may be interesting not only through their homology with the normal, i.e., nonpathological, human aging process, but also as an animal model of dementia.

\section{References}

Benson, D. F. (1983) Alterations in glucose metabolism in Alzheimer's disease. In Banbury Report 15: Biological Aspects of Alzheimer's Disease, R. Katzman, ed., pp. 309-316, Cold Spring Harbor Laboratory, Cold Spring Harbor, NY.

Birren, J. E., J. Botwinick, A. D. Weiss, and D. F. Morrison (1963) Interrelations of mental and perceptual tests given to healthy elderly men. In Human Aging. A Biological and Behavioral Study, J. E. Birren, ed., pp. 143-156, U. S. Government Printing Office, Washington, D. C.

Campbell, B. A., E. E. Krauter, and J. E. Wallace (1980) Animal models of aging: Sensory-motor and cognitive function in the aging rat. In Psychology of Aging: Problems and Prospectives, D. G. Stein, ed., pp. 201-226, Elsevier-North Holland Publishing Co., Amsterdam.

Davis, P., R. Katzman, R. D. Terry (1980) Reduced somatostatin-like immunoreactivity in cerebral cortex from cases of Alzheimer's disease and Alzheimers senile dementia. Nature (Lond.) 288: 279-280.

deLeon, M. J., S. H. Harris, A. E. George, B. Reisberg, D. R. Christman, I. I. Kricheff, and A. P. Wolf (1983) Computed tomography and positron emission transaxial tomography evaluation of normal aging and Alzheimer's Disease. J. Cerebr. Blood Flow Metab. 3: 391-394.

Frackowiak, R. S. J., and J. M. Gibbs (1983) The pathophysiology of Alzheimer's Disease studied with positron emission tomography. In Banbury Report 15: Biological Aspects of Alzheimer's Disease, R. Katzman, ed. pp. 317-328, Cold Spring Harbor Laboratory, Cold Spring Harbor, NY.

Gage, F. H., S. B. Dunnett, A. Björklund, and U. Stenevi (1982) Functional changes following embryonic grafts to the deafferented hippocampal formation. Soc. Neurosci. Abstr. 8: 1979.

Gage, F. H., S. B. Dunnett, U. Stenevi, and A. Björklund (1984a) Aged rats: Recovery of motor impairments by intrastriatal nigral grafts. Science 221: 966-969.

Gage, F. H., S. B. Dunnett, and A. Björklund (1984b) Spatial learning and motor deficits in aged rats. Neurobiol. Aging 5: 43-48.

Ingvar, D. W., A. Brun, B. Hagberg, and L. Gustafson (1977) Regional cerebral blood flow in the dominant hemisphere in confirmed cases 
of Alzheimer's disease, Pick's disease and multi-infarct dementia: Relationship to clinical symptomatology and neuropathological findings. In Alzheimer's Disease: Senile Dementia and Related Disorders, R. Katzman, P. Davis, and R. D. Terry, eds., pp. 441-451, Raven Press, New York.

Kety, S. S. (1956) Human cerebral blood flow and oxygen consumption as related to ageing. Res. Publ. Assoc. Res. Nerv. Ment. Dis. 35: 3145 .

Kolb, B., R. S. Sutherland, and I. Q. Whishaw (1983) A comparison of the contributions of the frontal and parietal association cortex to spatial localization in rats. Behav. Neurosci. 97: 13-27.

Kuhl, D. E., E. J. Metter, W. H. Riege, and M. E. Phelps (1982) Effects of human aging on patterns of local cerebral glucose utilization determined by the $\left({ }^{18} \mathrm{~F}\right)$ fluorodeoxyglucose method. J. Cerebr. Blood Flow Metab. 2: 163-171.

London, E. D., S. M. Nespor, M. Ohata, and S. I. Rapoport (1981) Local cerebral glucose utilization during development and aging of the Fischer-344 rat. J. Neurochem. 37: 217-221.

Marshall, J. F., and N. Berrios (1979) Movement disorders of aged rats: Reversal by dopamine receptor stimulation. Science 206:477-479.

Martinez, J. L., C. Petty, R. B. Messing (1982) Regional brain uptake of 2-deoxyglucose following training in a discriminated $\mathrm{Y}$-maze avoidance task. J. Comp. Physiol. Psychol. 96: 721-724.

Matsunami, K., and K. Kubota (1983) Radioactive deoxyglucose uptake into the prefrontal cortex during a delayed response task of the monkey. Neurosci. Lett. 36: 329-333.

Matsunami, K., T. Kageyama, and K. Kubota (1981) Radioactive zdeoxy-D-glucose incorporation into the prefrontal and premolor cortex of the monkey performing a forelimb movement. Neurosci. Lett. 26: $37-41$.

Morris, R. G. M. (1981) Spatial localization does not require the presence of local cues. Learn. Motiv. 12: 239-260.

Morris, R. G. M., P. Garrud, J. N. P. Rawlins, and J. O'Keefe (1982) Place navigation impaired in rats with hippocampal lesions. Nature (Lond.) 297: 681-683.

Perry, E. K., L. Tomlinson, G. Blessed, K. Bergmann, P. H. Gibson, and R. H. Perry (1978) Correlation of cholinergic abnormalities with senile plaques and mental test scores in senile dementia. Br. Med. J. 2: 1457.

Rapoport, S. I. (1983) Session 6: Summary and discussion. In Banbury Report 15: Biological Aspects of Alzheimer's Disease, R. Katzman, ed., pp. 329-334, Cold Spring Harbor Laboratory, Cold Spring Iarbor, NY.

Rapoport, S. I., R. Duara, E. D. London, R. A. Margolin, M. Schwartz,
N. R. Cutler, M. Partanen, and N. L. Shinohara (1983) Glucose metabolism of the aging nervous system. In Aging of the Brain, D. Samuel, ed., pp. 111-121, Raven Press, New York.

Schuier, F., F. Orzi, S. Suda, C. Kennedy, and L. Sokoloff (1981) The lumped constant for the $\left[{ }^{14} \mathrm{C}\right]$ deoxyglucose method in hyperglycemic rats. J. Cerebr. Blood Flow Metab. 1 (Suppl. 1): S63.

Schwartzman, R. J., J. Greenberg, M. Reivich, K. J. Klose, and G. M. Alexanders (1981) Functional metabolic mapping of a conditioned motor task in primates utilizing $2-\left[{ }^{14} \mathrm{C}\right]$-deoxyglucose. Exp. Neurol. 72: $153-163$

Shimada, M., T. II. Murakami, T. Imahayashi, and H. S. Ozaki (1983) Local cerebral alterations in $\left[{ }^{14} \mathrm{C}-2\right]$ deoxyglucose uptake following memory formation. J. Anat. 136: 751-759.

Smith, C. B., and L. Sokoloff (1982) Age related changes in local glucose utilization in the brain. Exp. Brain Res. Suppl. 5: 75-85.

Smith, C. B., C. Goochee, S. I. Rapoport, and L. Sokoloff (1980) Effects of aging on local rates of cerebral glucose utilization in the rat. Brain 103: $351-365$.

Sokoloff, L. (1966) Cerebral circulating and metabolic changes associated with aging. Res. Publ. Assoc. Res. Nerv. Ment. Dis. 41: 237254.

Sokoloff, L. (1981) The relationship between function and energy metabolism: Its use in the localization of functional activity in the nervous system. Neurosci. Res. Program Bull. 19: 159-210.

Sokoloff, L., M. Reivich, C. Kennedy, M. H. Des Rosiers, C. S. Patlak, K. D. Pettigrew, O. Sakurada, and M. Shinohara (1977) The $\left[{ }^{14} \mathrm{C}\right] 2$ deoxyglucose method for the measurement of local cerebral glucose utilization: Theory, procedure, and normal values in the conscious and anesthetized albino rat. J. Neurochem. 28: 897-916.

Suda, S., M. Shinohara, M. Miyaoka, C. Kennedy, and L. Sokoloff (1981) Local cerebral glucose utilization in hypoglycemia. J. Cerebr. Blood Flow Metab. 1 (Suppl. 1): S62.

Takai, H., W. R. Fredericks, E. D. London, and S. I. Rapoport (1983) Cerebral blood flow and oxidative metabolism in conscious Fischer 344 rats of different ages. J. Neurochem. 40: 801-805.

Turner, F. D., and F. H. Gage (1982) Measurement in psychobiology: Dynamics of the unconditioned response. Physiol. Behav. 29: 957960.

Wallace, J. E., E. E. Krauter, and B. A. Campbell (1980) Animal models of declining memory in the aged: Short-term and spatial memory in the aged rat. J. Gerontol. 35: 355-363.

Whitehouse, P. J., D. L. Price, R. G. Struble, A. W. Clark, and M. R. DeLong (1982) Alzheimer's disease and senile dementia: Loss of neurons in the basal forebrain. Science 215: 1237-1239. 